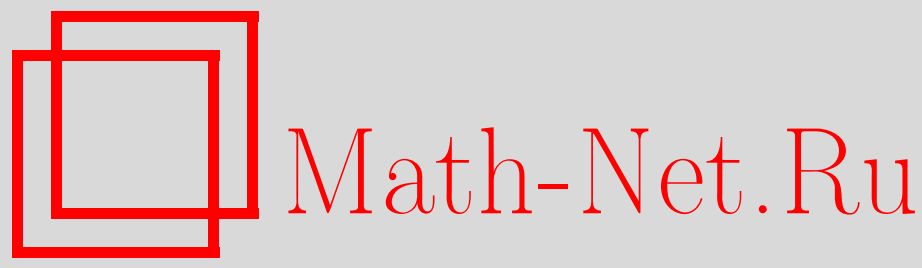

В. П. Маслов, Сжижение газа, подчиняющегося микроканоническому распределению, ТМ $\Phi, 2009$, том 160, номер 2, 331-332

DOI: https://doi.org/10.4213/tmf6401

Использование Общероссийского математического портала Math-Net.Ru подразумевает, что вы прочитали и согласны с пользовательским соглашением http://www . mathnet.ru/rus/agreement

Параметры загрузки:

IP : 52.6 .47 .48

26 апреля 2023 г., 10:53:06

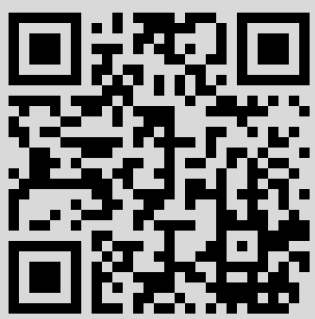




\section{СЖИЖЕНИЕ ГАЗА, ПОДЧИНЯЮЩЕГОСЯ МИКРОКАНОНИЧЕСКОМУ РАСПРЕДЕЛЕНИЮ}

На основе микроскопического распределения для идеального газа выводятся точные соотношения, приводящие к фазовому переходу первого рода.

Ключевые слова: бозе-конденсат, димеры, кластеры, перколяция.

Как известно, для невзаимодействующих частиц газа имеет место микроканоническое распределение вида

$$
\varepsilon_{1} \sum_{i=1}^{s} i^{l} N_{i}=\mathcal{E}, \quad \sum_{i=1}^{s} N_{i}=N
$$

где $\varepsilon_{1}$ - некоторая элементарная энергия (глубина потенциальной ямы в модели Леннарда-Джонса). При условии, что все варианты, отвечающие условию (1), равновероятны и $M=\mathcal{E} / \varepsilon_{1}$ - целое число, эта задача рассматривается в рамках теории распределений в аналитической теории чисел, так как $N \rightarrow \infty, s \rightarrow \infty, M \rightarrow \infty$ и $l=$ const. Однако, насколько известно автору, даже при $l=1, s=\infty$ в теории чисел она не была полностью решена. Пусть

$$
c_{\gamma}=\int_{0}^{\infty}\left(\frac{1}{\xi}-\frac{1}{e^{\xi}-1}\right) d \xi^{\gamma}, \quad b_{\gamma}=\int \frac{\xi d \xi^{\gamma}}{e^{\xi}-1}, \quad a_{\gamma}=\int \frac{d \xi^{\gamma}}{e^{\xi}-1},
$$

где $\gamma=\alpha / l$, а фрактальная коразмерность $\alpha$ зависит от $s$ и определяется из соотношения

$$
\alpha=l \lim \left(\frac{\ln (s / N)}{\ln (M / N)}\right),
$$

где $s, N, M$ стремятся к бесконечности.

Между величинами $s, l, M$ и $N$ возникают некоторые критические соотношения, в том числе величина $N_{\mathrm{cr}}$, определяющая переход в конденсатное состояние, для

* Московский государственный университет им. М. В. Ломоносова, Москва, Россия. E-mail: v.p.maslov@mail.ru 
различных фрактальных коразмерностей $\gamma$ имеет следующий вид:

$$
N_{\mathrm{cr}}(\gamma)= \begin{cases}c_{\gamma}^{1 / \gamma} b_{\gamma}^{-\gamma} M^{1 /(1+\gamma)}, & \gamma<1, \\ \frac{\sqrt{6}}{\pi} \sqrt{M} \ln N_{\mathrm{cr}}, & \gamma=1, \\ b_{\gamma} a_{\gamma}^{-1} M^{1+1 / \gamma}, & \gamma>1 .\end{cases}
$$

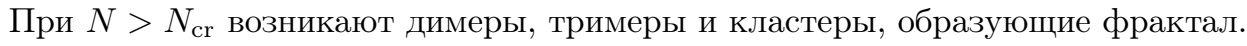

Рассмотрим случай, когда $l=2, \alpha=3$. Пусть $\kappa=0$. Тогда фактор сжимаемо-

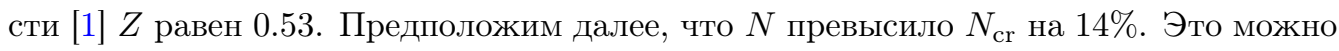
сделать, например, немного понизив температуру. Тогда образуется $7 \%$ димеров (критерий Кало). Это означает, что появились вкрапления, уменьшившие общий объем $V$ на 7\%. В результате фрактальная коразмерность $\alpha$ уменьшается, $\alpha<3$, а с ней уменьшается и $N_{\mathrm{cr}}(\alpha)(3)$ (см. [2]). Следовательно, появляются еще кластеры, и фрактальная коразмерность еще уменьшается, и так до коразмерности 2 включительно (т.е. до кластера фрактальной размерности, равной единице) идет лавинообразный процесс (цепная реакция) вплоть до $Z \approx \pi / 6 \ln N_{\text {сr }}(2) \approx 0.03$.

При увеличении давления и дальнейшем уменьшении размерности $(\alpha<2)$ величина $N_{\mathrm{cr}}(\alpha)$ лишь возрастает [3], а следовательно, плотность $\rho=N / V$ остается неизменной. Тем самым фактор сжимаемости увеличивается пропорционально увеличению давления. Следовательно, возникает фазовый переход первого рода (без метастабильных состояний) - перколяция от кластеров в газе мономеров к газовым нанопорам в жидкости.

Для реальных газов $\kappa \sim$ const $\frac{\ln N}{N}$. Ситуация идентичная, однако минимальное значение фактора сжимаемости возрастает до 0.2 .

Каноническое распределение, отвечающее (1), определено в работе [4], если положить $H(p)=\xi^{l}, d p=d \xi^{\gamma}$, а $\varphi(x)=\varphi\left(\xi^{l}\right)$.

Для объяснения фазовых переходов жидкость-твердое тело и газ-твердое тело идеальный газ мономеров определим как газ точек $(l=2, \alpha=3)$, идеальную жидкость - как вращающиеся димеры (несжимаемая жидкость) $(l=2, \alpha=2)$, идеальное твердое тело - как осциллирующие димеры $(l=1, \alpha=3)$. Поскольку часть энергии мономеров отдается димерам, размерность $\alpha$ уменьшается, и переход мономеров в конденсатное состояние можно рассматривать как переход поступательной энергии во вращательную и колебательную (см. [3]).

Фрактальная размерность смеси идеальной жидкости и идеального газа возрастает при увеличении давления до значения $\alpha=6$.

\section{Список литературы}

[1] А. И. Бурштейн, Молекулярная физика, Наука, Новосибирск, 1986.

[2] В. П. Маслов, В. Е. Назайкинский, Матем. заметки, 83:2 (2008), 232-263.

[3] V.P. Maslov, Threshold levels in Economics, arXiv: q-fin/ST0903.4783.

[4] В. П. Маслов, ТМФ, 159:2 (2009), 318-320. 